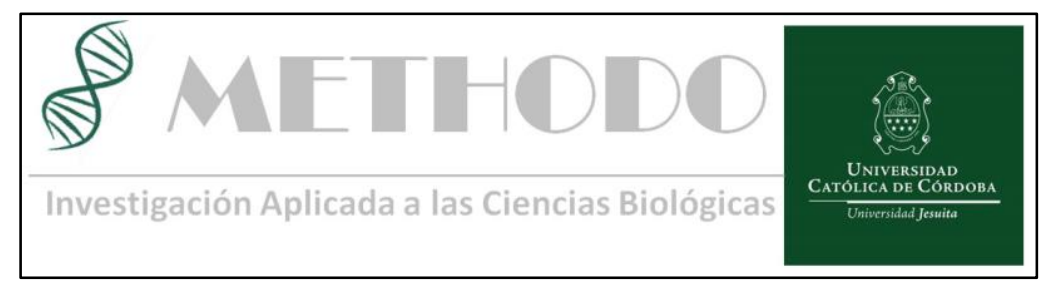

ARTICULO ORIGINAL Methodo 2017; 2(1):33-35 DOI: $10.22529 / \mathrm{me} .2017 .2(1) 07$

\title{
Relación entre sensibilización alérgica por pruebas cutáneas y grados clínicos de rinitis*
}

\section{Relationship between allergic sensitization by skin test and severity of rhinitis *}

${ }^{*}$ Resumen de Trabajo Presentado en las XIII Jornadas de Investigación de la Clínica Universitaria Reina Fabiola- Diciembre de 2016

Sosa Aguirre $\mathrm{A}^{1}$, Berardi $\mathrm{Y}^{1}$, Lozano $\mathrm{N}^{1}$, Ianiero $\mathrm{L}^{2}$, Saranz $\mathrm{R}^{1}$, Concari $\mathrm{E}^{1}$, Alegre $\mathrm{G}^{1}$, Lozano A. ${ }^{1}$

\section{RESUMEN Introducción:}

La rinitis alérgica $(R A)$ es una enfermedad inflamatoria de la mucosa nasal mediada por IgE. Existen controversias en los diferentes estudios acerca de la relación entre la gravedad de los síntomas de RA y la magnitud de la sensibilización medidas por pruebas cutáneas con alergenos.

\section{Objetivos:}

Relacionar la magnitud de sensibilización alérgica con los grados clínicos de la RA. Población y Método:

Estudio Observacional, analítico y transversal, que incluyó pacientes de ambos sexos, entre 6 y 18 años con diagnóstico de RA. Se clasificaron según el grado clínico establecido por Allergic rhinitis and its impact on asthma (ARIA). La sensibilización alérgica se midió a través de pruebas cutáneas por puntura con aeroalergenos y se determinó su magnitud según: PC1 (número de alérgenos positivos), PC2 (sumatoria de milímetros de pápulas) e Índice de atopía (relación entre PC2/PC1). Se dividieron según la presencia de monosensibilización y/o polisensibilización.

\section{Análisis Estadístico:}

Se realizó estadística descriptiva y analítica utilizando prueba de Wilcoxon Mann Withney y prueba de Kruskal Wallis. Se consideró un valor significativo a una $\mathrm{p}<0,05$.

\section{Resultados:}

Se incluyeron 156 pacientes con una mediana de edad de 11,18 años (5-18,66 años). No se encontró diferencia en los valores de PC1, PC2 ni en el índice de atopía entre los cuatro grados clínicos de rinitis $(p=0,3370, p=0,2222$ y $p=0,8774$ respectivamente). Tampoco se observó diferencia en el índice de atopia entre los pacientes monosensibilizados y polisensibilizados $(p=0,5493)$. Al comparar 
Sosa Aguirre A, Berardi Y, Lozano N, laniero L et al -

Relación entre sensibilización alérgica por pruebas cutáneas y grados clínicos de rinitis

la rinitis según la frecuencia no se encontró allergens), PC2 (sum of millimeters of diferencia en los valores de PC1, PC2 ni en papules) and index of atopy (relation el índice de atopía entre los grupos intermitente y persistente $(p=0,0810$; $p=0,0587$ y $p=0,5705)$. Con respecto a la gravedad de la rinitis no se encontró diferencia en los valores de PC1, PC2 ni en el índice de atopía entre los grupos leve y moderado/grave $(p=0,5693 ; p=0,1653$ y $p=0,4613)$.

\section{Conclusiones:}

Las pruebas cutáneas por puntura de lectura inmediata no son un método diagnóstico capaz de diferenciar los grados clínicos de rinitis..

\section{ABSTRAC}

\section{Introduction:}

Allergic rhinitis (RA) is an inflammatory disease of nasal mucosal mediated by $\lg \mathrm{E}$. There are controversies in the different studies about the relationship between the severity of RA symptoms and the magnitude of sensitization measured by skin tests with allergens.

\section{Objective:}

Relate the magnitude of allergic sensitization to the clinical grades of AR.

\section{Patients and Methods:}

In a observational, analytical and crosssectional study, we included patients aged 6 to 18 years with diagnosis of allergic rhinitis (RA). Were classified according to the clinical grade established by Allergic Rhinitis and its Impact on Asthma (ARIA) guide. Allergic sensitization was measured through skin prick test with aeroallergens and their magnitude was determined according to: PC1 (Number of positive 
Sosa Aguirre A, Berardi Y, Lozano N, laniero L et al -

Relación entre sensibilización alérgica por pruebas cutáneas y grados clínicos de rinitis

Palabras claves: RINITIS ALERGICA, PRUEBAS CUTANEAS POR PUNTURA, ARIA, AEROALERGENOS

(9)(1)()

Keywords: ALLERGIC RHINITIS, SKIN PRICK TEST, ARIA, AEROALLERGENS

'Universidad Católica de Córdoba, Argentina. Clínica Universitaria Reina Fabiola, Servicio de Alergia e Inmunología. Cátedra de Inmunología. Facultad de Medicina. ${ }^{2}$ Cátedra de Inmunología. Facultad de Medicina, Universidad Católica de Córdoba Correspondencia: Ana Gabriela Sosa Aguirre, Servicio de Alergia e Inmunología - Clínica Universitaria Reina Fabiola. Oncativo 1248 -X5004FHP- Córdoba, Argentina. e-mail: anag sosa@hotmail.com 